\title{
Actein inhibits cell proliferation and migration and promotes cell apoptosis in human non-small cell lung cancer cells
}

\author{
YUANYUAN ZHANG $^{1}$, JIANCHUN LIAN ${ }^{1}$ and XIAOWEI WANG ${ }^{2}$ \\ Departments of ${ }^{1}$ Laboratory Medicine and ${ }^{2}$ Oncology, Huai'an First People's Hospital, \\ Nanjing Medical University, Huai'an, Jiangsu 223300, P.R. China
}

Received March 4, 2017; Accepted November 10, 2017

DOI: $10.3892 / \mathrm{ol} .2017 .7668$

\begin{abstract}
Non-small cell lung cancer (NSCLC) is the leading cause of death in smokers and the most common cause for cancer mortality in both males and females in the United States. Predisposition of this malignancy to distant metastasis leads to poor prognosis; therefore, it is urgent to discover novel therapeutic agents for metastatic NSCLC. The present study aimed to investigate the effects of actein treatment on NSCLC cell growth and migration. Cell viability assays demonstrated that administration of actein markedly inhibited NSCLC cell proliferation in a dose- and time-dependent manner. Transwell assays demonstrated that actein treatment suppressed cell migration and invasion in two NSCLC cell lines, A549 and 95D. Furthermore, treatment with actein remarkably increased the activities of caspase-3 and -9 in NSCLC cells. The protein expression levels of cytoplasmic BCL2 apoptosis regulator (Bcl-2) and BCL2 associated X (Bax) were markedly decreased, while the protein expression levels of mitochondrial Bax, caspase-3, -9 and cytochrome $c$ were upregulated following actein treatment, as evidenced by western blot analysis. The present results demonstrated that actein inhibited cell proliferation and metastasis and promoted cell apoptosis in NSCLC cells, which indicated that actein administration might serve as a potential therapeutic strategy for the treatment of NSCLC in the clinic.
\end{abstract}

\section{Introduction}

Lung cancer was a rare disease at the beginning of the 20th century; however, nowadays, it is the leading cause of cancer-related deaths worldwide, with $17.2 \%$ morbidity in male and $11.6 \%$ in female smokers compared with 1.3 and $1.4 \%$ in non-smokers, respectively (1-3). Lung cancer can be divided

Correspondence to: Dr Yuanyuan Zhang, Department of Laboratory Medicine, Huai'an First People's Hospital, Nanjing Medical University, 6 Beijing Road West, Huai'an, Jiangsu 223300, P.R. China E-mail: yuanyuanzhang1234@163.com

Key words: actein, lung cancer, proliferation, migration, apoptosis into two categories: Non-small cell lung cancer (NSCLC) and small cell lung cancer (SCLC), of which the former one includes adenocarcinoma, squamous carcinoma, adenosquamous carcinoma and large cell lung cancer. Although efforts to develop novel therapies for NSCLC were made in the last decades, including chemotherapy and molecular targeted therapy, the five-yearsurvival rate remains $<5 \%$ for patients with metastatic NSCLC (4).

Perennial black cohosh is a common plant which is used by Native Americans to treat a variety of conditions, including arthritis, rheumatism muscle pain and dysmenorrhoea for centuries (5). It has also been a popular alternative for female menopausal symptoms (6). Recent studies indicate that black cohosh has minimal effects on tumor development. However, the extract of black cohosh has potential anticancer properties (7). The extract of black cohosh contains two major classes of secondary metabolites, triterpene glycosides and phenylpropanoids $(8,9)$. Of the $>42$ triterpene glycosides present in black cohosh, Actein has emerged to exert potent tumor inhibitory effects in human breast cancer (10). Although actein was demonstrated to exhibit other functions, such as prevention of oxidative damage to osteoblasts and regulation of lipid disorders, a major role of actein consists of its anticancer activity, particularly in breast cancer (11), where it was first reported that actein and a fraction of black cohosh inhibit breast cancer cells growth in vitro. Actein and fractions of black cohosh potentiate the antiproliferative effects of chemotherapy agents on human breast cancer cells, and its growth inhibitory effects are associated with activation of the stress response pathway and induction of calcium release (11). Of note, actein potentiatesdigitoxin's inhibitory effect on $\mathrm{Na}^{+}-\mathrm{K}^{+}$-ATPase activity and MDA-MB-453 breast cancer cell growth, indicating the potential of synergistic combination for cancer therapy (12).

An increasing number of studies have focused on the effects of actein in tumorigenesis. It has been reported that actein inhibits cell growth rates and promotes cell apoptosis in human osteosarcoma cells (13). In addition, actein increases the activity of glyoxalase I and levels of reduced glutathione by activating the transcriptional factor nuclear factor 2-related factor 2 (14). However, the specific role of actein in NSCLC remains largely unknown. The present study examined the effects of actein treatment on the cell survival rate, cell proliferation and migration of NSCLC cell lines. The results may 
provide novel information for the diagnosis and treatment of NSCLC in the clinic.

\section{Materials and methods}

Cell culture and reagents. NSCLC cell lines A549, H1975, SPC-A-1, H125 and 95D were commercially purchased from American Type Culture Collection (ATCC; Manassas, VA, USA) and cultured in the recommended medium supplied with 10\% fetal bovine serum (FBS) (both from (Gibco; Thermo Fisher Scientific, Inc., Waltham, MA, USA) in a 5\% $\mathrm{CO}_{2}$ atmosphere. Actein was purchased form Sigma-Aldrich (Merck KGaA, Darmstadt, Germany), prepared as a stock solution in methanol at a concentration of $120 \mu \mathrm{M}$ and diluted to desired doses based on experimental designs. Inhibitory rate was defined as (OD value in control group - OD value in experimental group $) /(\mathrm{OD}$ value in control group - OD value in blank control group) $\mathrm{x} 100 \%$.

Cell viability assay. Cell viability was assessed using an MTT assay. A total of $1 \times 10^{4}$ NSCLC cells were seeded in 96-well plates and treated with different concentrations of actein in triplicate. Following treatment (at different time intervals), $10 \mu 1$ of MTT $(5 \mu \mathrm{g} / \mathrm{ml})$ was mixed with the medium in each well and incubated for another $3 \mathrm{~h}$ at $37^{\circ} \mathrm{C}$ in the dark. Formazan crystals were dissolved in $100 \mu 1 \mathrm{DMSO}$ and the optical absorbance of each well was measured at $570 \mathrm{nM}$ with a TECAN plate-reader.

Colony formation assay. A549 and 95D cells in 6-well plates were treated with different concentrations of actein $(0,5,10$, 20,40 and $80 \mu \mathrm{M}$ ), and following $24 \mathrm{~h}$ of treatment, cells were seeded into 12-well plates (100 cells/well) in triplicate. The medium was changed every other day and following incubation for 10 days, the colonies were fixed with pre-iced methanol and stained with crystal violet. Colonies containing more than 50 cells were selected and counted as survivors under a Nikon microscope at a 200 magnification. The following formula was used to calculate the rate of colony formation: Colony formation rate $=$ (number of colonies/number of seeded cells) x $100 \%$.

Transwell assay. Cell migration and invasion were determined by transwell assays. First, cells were seeded in 6-well plates and treated with different doses of actein for $24 \mathrm{~h}$. Then, cells were resuspended in recommended medium without FBS and $1 \times 10^{4}$ A549 or 95D cells were placed in the upper chambers (Corning Inc., Corning, NY, USA) with a volume of $100 \mu 1$ per well. A total of $600 \mu 1$ of medium with $10 \%$ FBS was added in the bottom chambers. After incubation for $12 \mathrm{~h}$, cells were washed with PBS for three times, fixed with methanol for $10 \mathrm{~min}$, and stained with crystal violet for $5 \mathrm{~min}$. Cell migration was quantified by counting cells migrated across the filter towards the lower surface of the chamber. Five random fields were calculated under a Nikon microscope. For invasion assays, the upper surface of the chambers was pre-coated with Matrigel (BD Biosciences, Franklin Lakes, NJ, USA) for $6 \mathrm{~h}$ in a $37^{\circ} \mathrm{C}$ incubator prior to the assays. All experiments were repeated at least three times in triplicate.
Wound-healing assay. Wound-healing assays were performed by creating identical wound areas for the anchorage-dependent cells A549 and 95D with a $10 \mu 1$ sterile pipette tip. Cells were seeded into 6-well plates and incubated with different concentrations of actein for $24 \mathrm{~h}$. Next, cells were washed with PBS and a cross was scraped in the center of each well. Both A549 and 95D cells were washed with PBS and fresh serum-free medium was immediately added. Following $24 \mathrm{~h}$ growth, cells were observed and photographed under a Nikon microscope at a $\times 200$, magnification for each group ( 6 fields were counted/well and the experiment was repeated 3 times).

Cell apoptosis assay. Cell apoptosis was examined with Hoechst 33258 (Beyotime Institute of Biotechnology, Nanjing, China) according to the manufacturer's instructions. A total of $5 \times 10^{5}$ A549 and 95D cells were seeded into 12-well plates in triplicate and treated with different concentrations of actein $(0,5,10,20,40$ and $80 \mu \mathrm{M})$ for $48 \mathrm{~h}$. Afterwards, Hoechst 33258 was mixed with the medium in each well and incubated for $15 \mathrm{~min}$ at $37^{\circ} \mathrm{C}$. Fluorescence images were captured randomly ( 6 fields were counted per well and the experiment was repeated 3 times) with an inverted fluorescence microscope (Nikon Corporation, Tokyo, Japan). The percentage of apoptotic neurons were calculated with the following formula: Apoptotic rate $=$ (apoptotic cells/total cells) x $100 \%$.

Caspase activity assay. The activity of caspase- $3,-8$ and -9 was determined by caspase activity kits (Beyotime Institute of Biotechnology), according to the manufacturer's instructions. Briefly, A549 and 95D cells were treated with actein $(0,20$ and $40 \mu \mathrm{M}$ ) for $24 \mathrm{~h}$ and cell lysates were collected. Assays were performed in 96-well plates by incubating proteins of cell lysates and reaction buffers containing substrates for caspase-3, -8 and -9 , respectively. Following co-incubation for $4 \mathrm{~h}$, samples were measured with a TECAN plate-reader at an absorbance of $405 \mathrm{~nm}$. All experiments were repeated at least three times in triplicate.

Western blot analysis. A549 cells were treated with different doses of actein $(0,20$ and $40 \mu \mathrm{M})$ for $24 \mathrm{~h}$. Total proteins were extracted with NP-40 lysis buffer (Beyotime Institute of Biotechnology) and quantified by the standard BCA method (Thermo Fisher Scientific, Inc.). Equal amount of proteins from each sample $(50 \mu \mathrm{g})$ was separated with $12 \%$ SDS-PAGE and electroblotted onto nitrocellulose membranes. Following blocking with TBST/5\% milk for $1 \mathrm{~h}$ at room temperature, the membranes were incubated with primary antibodies at $4^{\circ} \mathrm{C}$ overnight. Primary antibodies against BCL2 apoptosis regulator (Bcl-2; 1:2,000, cat. no. ab32124) and BCL2 associated $X$ protein (Bax; 1:2,000, cat. no. ab32503) were both purchased from Abcam (Cambridge, UK). Primary antibodies against caspase-9 (1:2,000; cat. no. sc4704), caspase-3 (1:2,000; cat. no. sc1224), cytochrome $c$ (cyto $c ; 1: 2,000$, cat. no. sc8385) and GAPDH (1:1,000; cat. no. sc365062) and secondary antibodies were purchased from Santa Cruz Biotechnology, Inc. (1:1,500, cat. no. sc-2354; Dallas, TX, USA). The protein bands labeled with the antibodies were visualized using the SuperSignal West Pico Chemiluminescent Substrate Trial kit (Pierce 


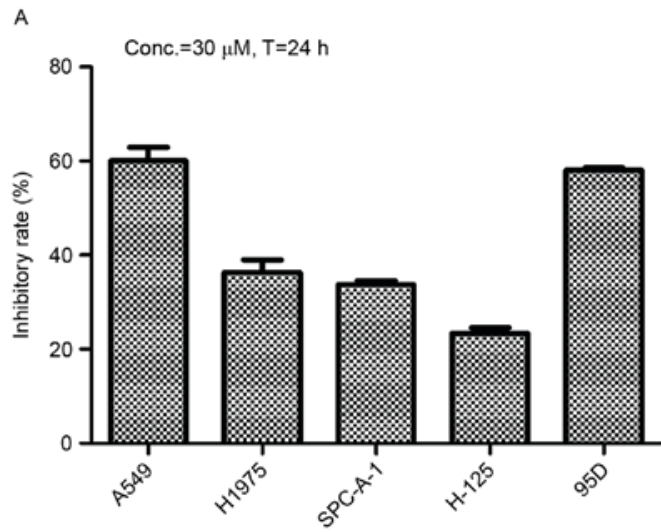

B
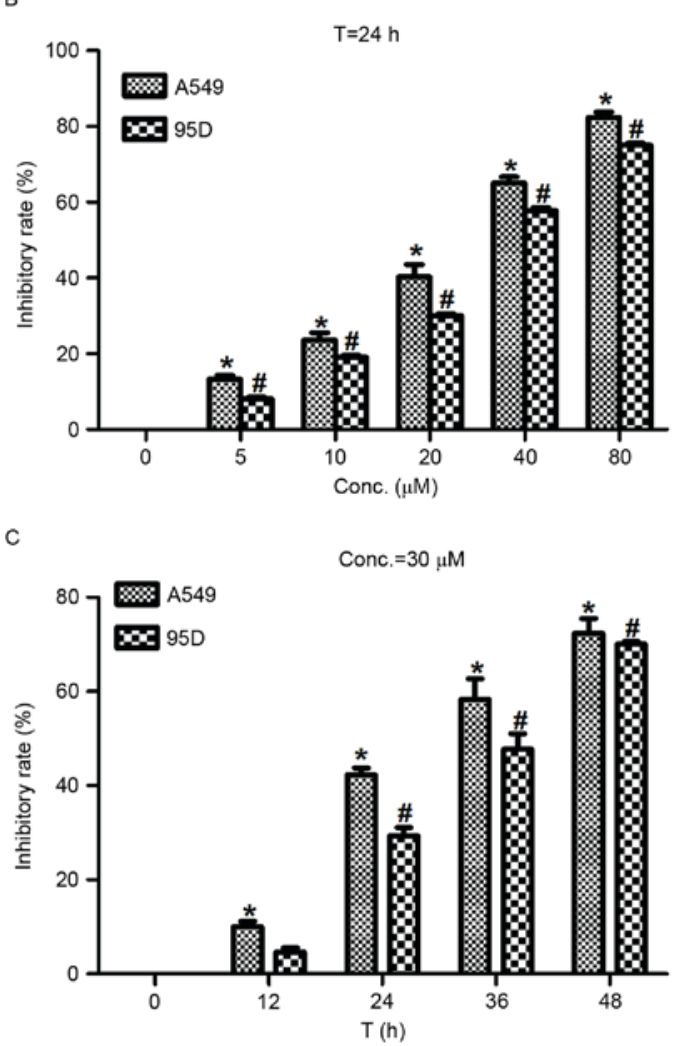

Figure 1. Actein inhibits cell viability in a dose- and time-dependent manner. (A) Cell viability of 5 different lung cancer cell lines was assessed following treatment with $30 \mu \mathrm{M}$ actein for $24 \mathrm{~h}$. (B) cell viability of A549 and 95D was examined following treatment with different doses of actein $(0,5,10$, 20, 40 and $80 \mu \mathrm{M}$ ) for $24 \mathrm{~h}$. (C) cell viability of A549 and 95D was examined following treatment with $30 \mu \mathrm{M}$ actein for different time periods $(0$, $12,24,36$ and 48 h). ${ }^{*} \mathrm{P}<0.05$, compared with untreated A549 cells; ${ }^{*} \mathrm{P}<0.05$, compared with untreated $95 \mathrm{D}$ cells.

Protein Biology; Thermo Fisher Scientific, Inc.). Images were obtained using the ChemiDoc XRS system with Quantity One software (Bio-Rad Laboratories, Inc., Hercules, CA, USA). Protein expression was analyzed using BandScan version 5.0 software (Glyko, Inc., Novato, CA, USA).

Statistical analysis. All data were presented as the mean \pm standard deviation. Eachexperiment was repeated atleast three times in triplicate, except if otherwise stated. Statistical evaluation was performed using Student's t-test or one-way analysis of variance followed by Student-Newman-Keuls test with SAS (version 9.2; SAS Institute Inc., Cary, NC, USA).
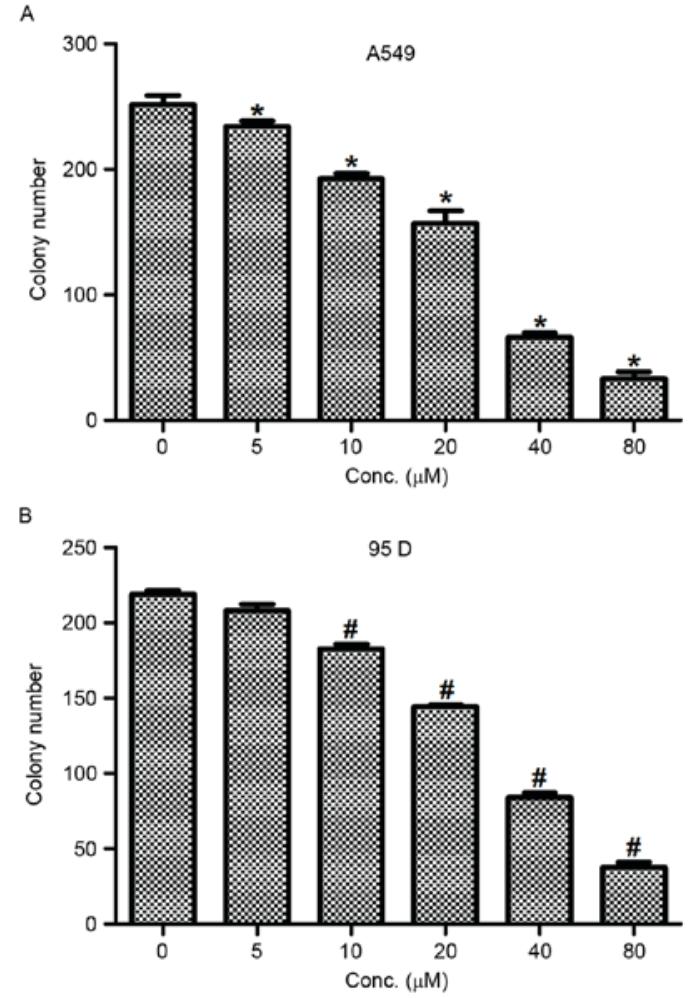

Figure 2. Actein inhibits colony formation in both A549 and 95D cells. (A) A549 and (B) 95D cells were treated with different doses of actein $(0,5$, $10,20,40$ and $80 \mu \mathrm{M})$ for $24 \mathrm{~h}$ and colony formation was measured. ${ }^{*} \mathrm{P}<0.05$ compared with untreated A549 cells; ${ }^{\#} \mathrm{P}<0.05$, compared with untreated $95 \mathrm{D}$ cells.

$\mathrm{P}<0.05$ was considered to indicate a statistically significant difference.

\section{Results}

Actein inhibits cell viability in NSCLC cells. To test the effects of actein administration on NSCLC cell proliferation, NSCLC cell lines A549, H1975, SPC-A-1, H-125 and 95D were cultured and treated with actein $(30 \mu \mathrm{M}) 24 \mathrm{~h}$ prior to cell viability assays. The results demonstrated that cell viability rates in all these five cell lines were reduced following actein administration, especially in A549 cells and 95D cells (Fig. 1A). Therefore, the A549 and 95D cells were selected for subsequent experiments. Next, the 2 cell lines were treated with different doses of actein at diverse time intervals. Fig. 1B and C illustrate that actein significantly suppressed cell viability in a dose- and time-dependent manner, compared with control cells. The lethal dose 50 value $\left(\mathrm{LD}_{50}\right)$ was approximate 20 and $30 \mu \mathrm{M}$, respectively, for A549 and 95D cells $24 \mathrm{~h}$ following actein administration (Fig. 1B). The inhibitory rate was progressively increased with extended treatment time for both cell lines stimulated with $30 \mu \mathrm{M}$ actein (Fig. 1C). These data revealed that actein decreased the cell viability of NSCLC cell lines in a dose- and time-dependent manner.

Actein suppresses cell colony formation in NSCLC cells. To further assess the inhibitory effects of actein in NSCLC, a colony formation assay was conducted in A549 and 95D cells. 
A

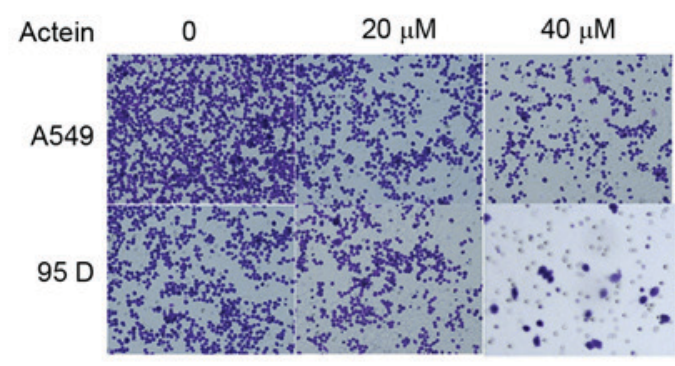

C

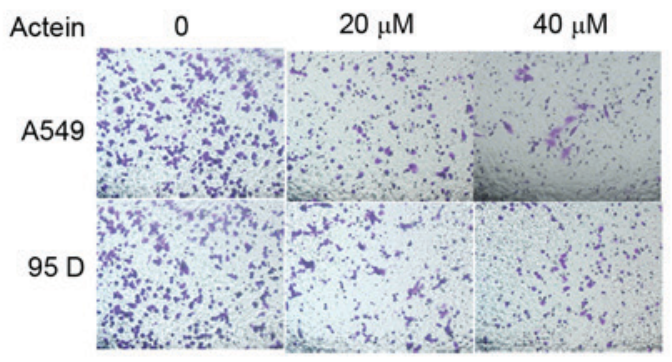

B

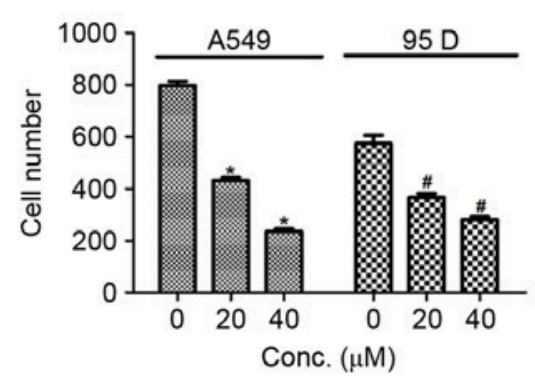

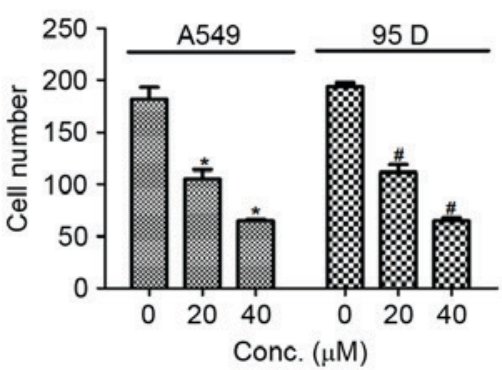

$\mathrm{E}$

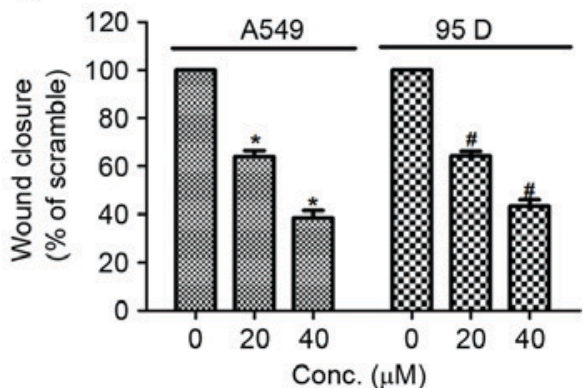

Figure 3. Actein inhibits cell migration and invasion in lung cancer cell lines. (A) Representative images of cell migration assays (magnification, x400). (B) Quantification of migrated cell numbers in A549 and 95D cells treated with actein. (C) Representative images of cell invasion assays, $\mathrm{x} 400$ magnification. (D) Quantification of invaded cell numbers in A549 and 95D cells treated with actein. (E) Quantification of wound healing assays for A549 and 95D cells treated with actein. ${ }^{*} \mathrm{P}<0.05$, compared with untreated A549 cells; ${ }^{*} \mathrm{P}<0.05$, compared with untreated $95 \mathrm{D}$ cells.

Cells were treated with different doses of actein $(0,5,10,20$, 40 and $80 \mu \mathrm{M})$. In control untreated A549 cells, 250 colonies were visualized; however, only 160 and 35 colonies were visualized when A549 cells were treated with 20 and $80 \mu \mathrm{M}$ actein, respectively (Fig. 2A). In 95D cells, the colony numbers were also dramatically decreased from 230 in the control group to 35 in the $80 \mu \mathrm{M}$ actein treated group (Fig. 2B). These data demonstrated that actein inhibited colony formation, jointly leading to the conclusion that actein treatment suppressed cell proliferation in NSCLC cell lines.

Actein inhibits cell migration and invasion in NSCLC cells. Next, transwell assays were performed to explore the effects of actein in cell migration and invasion in human NSCLC cells. As illustrated in Fig. 3A and B, the number of cells migrated to the lower surface of the chamber was decreased by 50 and $75 \%$ when A549 cells were treated with 20 and $40 \mu \mathrm{M}$ actein respectively, compared with control. Significant decreases in cell migration were also observed in $95 \mathrm{D}$ cells upon actein administration. Cell invasion ability was also measured by using Transwell chambers that was pre-coated with Matrigel for $6 \mathrm{~h}$. The results demonstrated that the number of cells invaded through the membrane was significantly decreased in A549 cells treated with actein, in a dose-dependent manner (Fig. 3C and D). Similarly, the invasion abilities of 95D cells treated with 20 or $40 \mu \mathrm{M}$ actein were decreased compared with control (Fig. 3C and D). Furthermore, wound-healing assays were performed to further confirm the effects of actein on NSCLC cell migration. A549 and 95D cells were treated with 20 or $40 \mu \mathrm{M}$ actein prior to scraping a cross in each well. Fig. 3E illustrates that the rates of wound closure were significantly decreased for both actein-treated cell lines compared with untreated controls. These effects were also dose-dependent. The present data suggested that actein suppressed cell migration and invasion in vitro.

Actein increases NSCLC cell apoptosis in a dose-dependent manner. Actein was reported to exhibit a significant regulatory role in cell apoptosis in melanoma cells (15). Thus, the effects of actein in NSCLC cell apoptosis were examined in the present study with Hoechst 33258 staining analysis. Following exposure to various concentrations $(0,5,10,20$, 40 and $80 \mu \mathrm{M}$ ) of actein, the cell nuclei of A549 and 95D cells were observed to be crushed and fragmented with Hoechst 33258 staining. The results demonstrated that cell apoptosis rates were increased by $5,12,18,23$ and $30 \%$ in A549 cells treated with 5, 10, 20, 40 and $80 \mu \mathrm{M}$ actein respectively (Fig. 4A). Similar results were observed in 95D cells. Apoptosis rates were increased from $3 \%$ in control cells to $35 \%$ in the $80 \mu \mathrm{M}$ actein-treated cells (Fig. 4B). Next, the relative caspase activity was explored in cells that were treated with actein $(0,20$ and $40 \mu \mathrm{M})$. It was demonstrated that the activities of caspase- 3 and caspase- 9 were significantly increased by actein treatment in a dose-dependent manner, while the activity of caspase- 8 remained unchanged in both NSCLC cell lines (Fig. 4C and D).

Finally, the expression of apoptosis-related proteins in A549 cells was examined by western blot analysis. As illustrated in Fig. 5, when A549 cells were treated with various doses of actein $(0,20$ and $40 \mu \mathrm{M})$, the protein expression levels of Bcl-2 were decreased as the concentration of actein increased. In addition, the protein expression levels of mitochondrial Bax, cleaved caspase-3, -9 and cytoplasmic cyto $c$ were significantly upregulated following actein treatment in 


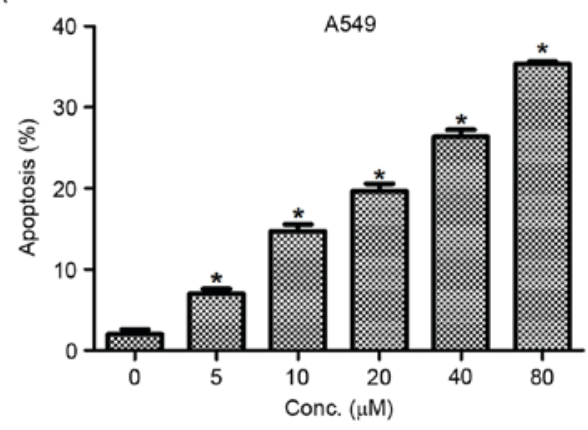

C

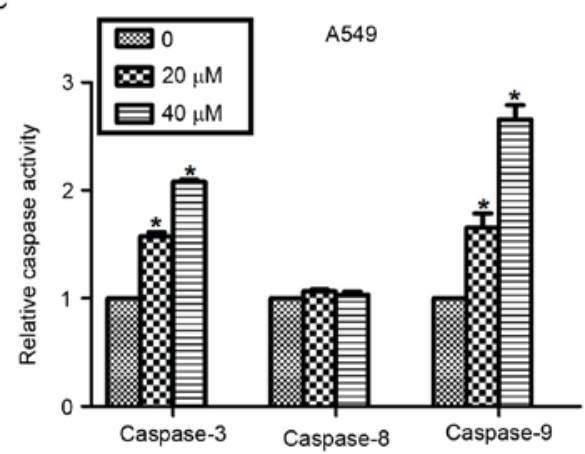

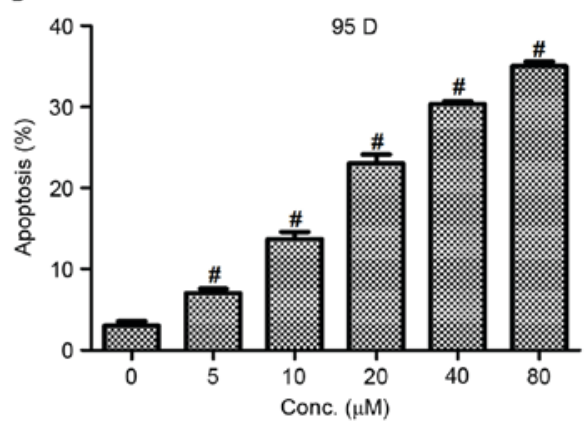

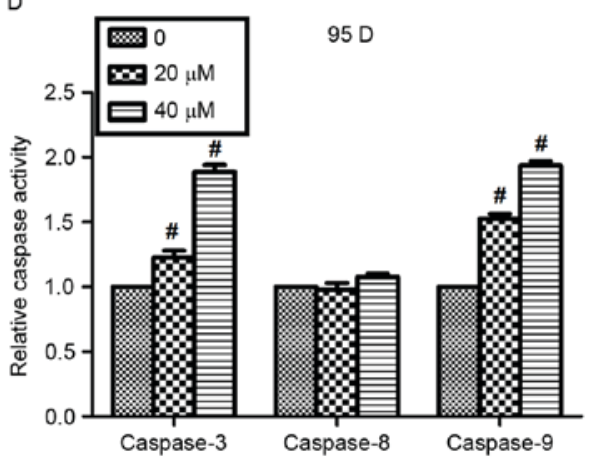

Figure 4. Actein promotes cell apoptosis in lung cancer cells. (A) A549 and (B) 95D cells were treated with different concentrations of actein prior to Hoechst 33258 staining for calculation of the apoptosis rate. (C) A549 and (D) 95D cells were treated with different concentrations of actein and then examined for caspase-3, -8 and -9 activities. ${ }^{*} \mathrm{P}<0.05$, compared with untreated A549 cells; ${ }^{*} \mathrm{P}<0.05$, compared with untreated 95D cells.

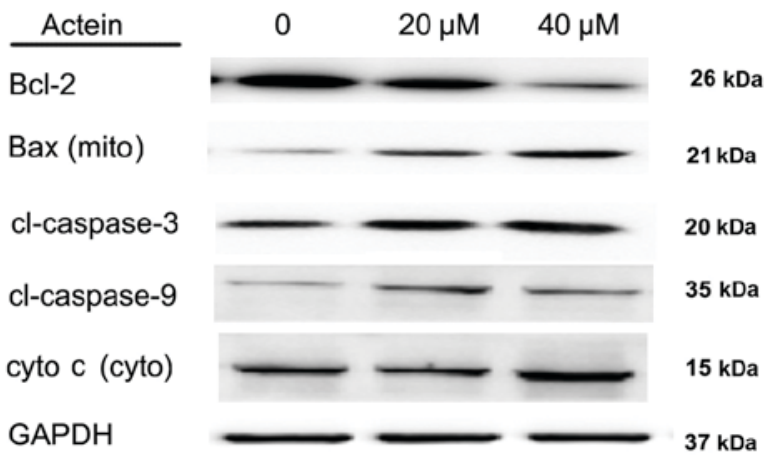

Figure 5. Actein treatment alters expression of apoptosis-related proteins in A549 cells. A549 cells were treated with different doses of actein $(0,20$ and $40 \mu \mathrm{M})$ and total proteins in each group were subjected to western blot analysis. Protein levels of Bcl-2 were decreased, while levels of Bax (mito), cl-caspase-3, -9, cyto $c$ (cyto) were increased following actein treatment in a dose-dependent manner. Bcl-2, BCL2 apoptosis regulator; Bax, BCL2 associated X; cyto, cytoplasmic; mito, mitochondrial; cl, cleaved; cyto $c$, cytochrome $c$.

a dose-dependent manner (Fig. 5). Together with Fig. 4, the present results suggested that actein promoted NSCLC cell apoptosis in a dose-dependent manner.

\section{Discussion}

NSCLC is a life-threatening cancer with an obvious etiology and various pathogenic factors. In the future, it is likely to become the world's leading cause of cancer-related deaths despite worldwide efforts to control the etiology of the disease. The identification of novel genetic biomarkers and effective therapeutic agents has been a huge step forward to understanding the tumorigenesis, diagnosis and treatment of NSCLC (16-18). However, further studies are required in order to increase the five-year survival rate for patients suffering from NSCLC.

The present study demonstrated the inhibitive effects of actein on NSCLC cell proliferation and migration in vitro. Actein suppressed cell viability in a dose- and time-dependent manner. Furthermore, the colony formation assay confirmed that, upon actein administration, NSCLC cell lines A549 and 95D displayed reduced abilities to form colonies, which suggested an inhibited proliferation property. Transwell assays revealed that actein treatment suppressed cell migration and invasion in NSCLC cells. Since cell proliferation and migration are hallmarks of cancer, these data suggest that actein might function as a potential therapeutic agent against NSCLC through its strong growth inhibitory effects. Furthermore, lower and higher concentrations of actein were explored in the present preliminary study. It was demonstrated that the cell proliferative rate and invasive capacity of both A549 and 95D cells were suppressed following actein treatment, even in a lower concentration $(30 \mu \mathrm{M})$. Therefore, the inhibitory effects of actein on cell proliferation and invasion were synchronous. The detailed mechanism underlying the inhibitory effects of actein remains unknown and this will be the focus of future studies.

The induction of apoptosis can be divided into two categories: Intrinsic and extrinsic pathways. The initiation of the former is associated with the pro-apoptotic factors Bax and BCL2 associated agonist of cell death (Bad), and it results in increased permeability of the mitochondria membrane, loss of membrane potential and release of cyto $c$ into the cytosol (19). 
Cyto $c$ serves a regulatory role since it precedes morphological changes associated with apoptosis. Cyto $c$ binds to apoptotic protease activating factor- 1 and then pro-caspase- 9 to form a protein complex called apoptosome, the role of which is to cleave pro-caspase to its active form of caspase- 9 and then in turn activate caspase-3 (20). In the present study, the apoptosis rate following actein administration was explored and the results demonstrated thatactein could positively promote cell apoptosis in NSCLC cells. Furthermore, the expression levels of mitochondrial Bax, caspase-3, -9 and cyto $c$ were all upregulated in actein-treated A549 cells in a dose-dependent manner, while actein inhibited the protein levels of Bcl-2 and cytoplasmic Bax. Bcl-2 and Bax both belong to the BCL2 family, the former of which is anti-apoptotic while the latter one is proapoptotic. When apoptosis occurs, the proapoptotic homodimers are essential to make the mitochondrial membrane permeable for the release of caspase activators including cyto $c$ (21), resulting in decreased expression of cytoplasmic Bax and increased mitochondrial Bax during apoptosis. Since induction of apoptosis is always associated with proliferation inhibition, it is hypothesized that actein may inhibit cell proliferation and migration through the apoptosis pathway. However, the detailed mechanisms and signaling processes by which actein affects the apoptosis pathways remain to be elucidated.

In conclusion, the present study revealed the growth inhibitory effectof actein in NSCLC cells, which was related with cell apoptosis. Theseresults might provide new clues for the treatment of NSCLC in the clinic. Actein may serve as a potential therapeutic agent against NSCLC.

\section{References}

1. Villeneuve PJ and Mao Y: Lifetime probability of developing lung cancer, by smoking status, Canada. Can J Public Health 85: 385-388, 1994

2. Torre LA, Bray F, Siegel RL, Ferlay J, Lortet-Tieulent J and Jemal A: Global cancer statistics, 2012. CA Cancer J Clin 65: 87-108, 2015.

3. Jemal A, Bray F, Center MM, Ferlay J, Ward E and Forman D: Global cancer statistics. CA Cancer J Clin 61: 69-90, 2011.

4. Chen W, Zheng R, Zeng H and Zhang S: Epidemiology of lung cancer in China. Thorac Cancer 6: 209-215, 2015.

5. McKenna DJ, Jones K, Humphrey S and Hughes K: Black cohosh: Efficacy, safety, and use in clinical and preclinical applications. Altern Ther Health Med 7: 93-100, 2001.

6. Einbond LS, Su T, Wu HA, Friedman R, Wang X, Ramirez A, Kronenberg F and Weinstein IB: The growth inhibitory effect of actein on human breast cancer cells is associated with activation of stress response pathways. Int J Cancer 121: 2073-2083, 2007.
7. Lee YS and Choi EM: Actein isolated from black cohosh promotes the function of osteoblastic MC3T3-E1 cells. J Med Food 17: 414-423, 2014

8. Einbond LS, Soffritti M, Esposti DD, Park T, Cruz E, Su T, Wu HA, Wang X, Zhang YJ, Ham J, et al: Actein activates stress- and statin-associated responses and is bioavailable in Sprague-Dawley rats. Fundam Clin Pharmacol 23: 311-321, 2009.

9. Einbond LS, Shimizu M, Xiao D, Nuntanakorn P, Lim JT, Suzui M, Seter C, Pertel T, Kennelly EJ, Kronenberg F and Weinstein IB: Growth inhibitory activity of extracts and purified components of black cohosh on human breast cancer cells. Breast Cancer Res Treat 83: 221-231, 2004.

10. Einbond LS, Shimizu M, Nuntanakorn P, Seter C, Cheng R, Jiang B, Kronenberg F, Kennelly EJ and Weinstein IB: Actein and a fraction of black cohosh potentiate antiproliferative effects of chemotherapy agents on human breast cancer cells. Planta Med 72: 1200-1206, 2006.

11. Einbond LS, Mighty J, Redenti S and Wu HA: Actein induces calcium release in human breast cancer cells. Fitoterapia 91: 28-38, 2013.

12. Einbond LS, Shimizu M, Ma H, Wu HA, Goldsberry S, Sicular S, Panjikaran M, Genovese G and Cruz E: Actein inhibits the $\mathrm{Na}^{+}-\mathrm{K}^{+}$-ATPase and enhances the growth inhibitory effect of digitoxin on human breast cancer cells. Biochem Biophys Res Commun 375: 608-613, 2008.

13. Chen Z, Wu J and Guo Q: Actein inhibits cell proliferation and migration in human osteosarcoma. Med Sci Monit 22: 1609-1616, 2016.

14. Suh KS, Chon S and Choi EM: Actein protects against methylglyoxal-induced oxidative damage in osteoblastic MC3T3-E1 cells. J Sci Food Agric 97: 207-214, 2017.

15. Du J, Lu X, Long Z, Zhang Z, Zhu X, Yang Y and Xu J: In vitro and in vivo anticancer activity of aconitine on melanoma cell line B16. Molecules 18: 757-767, 2013.

16. Lee PN and Hamling J: The relation between smokeless tobacco and cancer in Northern Europe and North America. A commentary on differences between the conclusions reached by two recent reviews. BMC Cancer 9: 256, 2009.

17. Hackshaw AK, Law MR and Wald NJ: The accumulated evidence on lung cancer and environmental tobacco smoke. BMJ 315: 980-988, 1997.

18. King PT: Inflammation in chronic obstructive pulmonary disease and its role in cardiovascular disease and lung cancer. Clin Transl Med 4: 68, 2015.

19. Spencer SL and Sorger PK: Measuring and modeling apoptosis in single cells. Cell 144: 926-939, 2011.

20. Dejean LM, Martinez-Caballero S and Kinnally KW: Is MAC the knife that cuts cytochrome $\mathrm{c}$ from mitochondria during apoptosis? Cell Death Differ 13: 1387-1395, 2006.

21. Murphy KM, Ranganathan V, Farnsworth ML, Kavallaris M and Lock RB: Bcl-2 inhibits Bax translocation from cytosol to mitochondria during drug-induced apoptosis of human tumor cells. Cell Death Differ 7: 102-111, 2000. 